\title{
Effect of the release from mechanical stress on osteoclastogenesis in RAW264.7 cells
}

\author{
KENJIRO SHIBATA $^{1,2}$, YOSHITAKA YOSHIMURA ${ }^{2}$, TAKASHI KIKUIRI ${ }^{3}$, TOMOKAZU HASEGAWA ${ }^{4}$, \\ YUMI TANIGUCHI $^{3}$, YOSHIAKI DEYAMA ${ }^{2}$, KUNIAKI SUZUKI ${ }^{2}$ and JUNICHIRO IIDA ${ }^{1}$ \\ Departments of ${ }^{1}$ Orthodontics, ${ }^{2}$ Molecular Cell Pharmacology and ${ }^{3}$ Pediatric Dentistry, Hokkaido \\ University Graduate School of Dental Medicine, Kita-ku, Sapporo 060-8586; ${ }^{4}$ Department of \\ Pediatric Dentistry, School of Dentistry, Iwate Medical University, Morioka 020-8505, Japan
}

Received November 12, 2010; Accepted December 30, 2010

DOI: $10.3892 / \mathrm{ijmm} .2011 .675$

\begin{abstract}
The effects of mechanical stress release on osteoclastogenesis may be as important as those of mechanical stress application. However, the direct effects of mechanical stress on the behavior of osteoclasts has not been thoroughly investigated and there is limited information on the results of the release from mechanical stress. In this study, the effects of mechanical stress application and its release on osteoclast differentiation were examined. The number of tartrate-resistant acid phosphatase (TRAP)-positive multinucleated osteoclasts derived from RAW264.7 cells were measured and the expression of osteoclast differentiation genes, which was altered in response to the release from mechanical stress according to the Flexercell tension system was evaluated by real-time PCR. Osteoclast differentiation and fusion were suppressed by mechanical stress application and were rapidly induced after mechanical stress release. The mRNA expression of the osteoclast specific genes, TRAP, matrix metalloproteinase-9 (MMP-9), cathepsin-K (cath-k), calcitonin receptor (CTR), ATPase $\mathrm{H}^{+}$transporting vacuolar proton pump member I (ATP6i), chloride channel-7 (ClC7) and dendritic cell-specific transmembrane protein (DC-STAMP) was decreased with mechanical stress application, and increased up to $48 \mathrm{~h}$ after the release from it. These alterations in gene mRNA expression were associated with the number of osteoclasts and large osteoclasts. Inducible nitric oxide synthetase (iNOS) mRNA was increased with mechanical stress and decreased after its release. Nitric oxide (NO) production was increased with mechanical stress. Nuclear factor of activated $\mathrm{T}$ cells cytoplasmic (NFATc) family mRNAs were not altered with mechanical stress, but were up-regulated up to $48 \mathrm{~h}$ after the release from it. These findings indicate that the suppression of osteoclast differentia-
\end{abstract}

Correspondence to: Dr Yoshitaka Yoshimura, Hokkaido University Graduate School of Dental Medicine, Kita 13 Nishi 7, Kita-ku, Sapporo 060-8586, Japan

E-mail: yoshi@den.hokudai.ac.jp

Key words: mechanical stress, osteoclast, nitric oxide tion and fusion induced by mechanical stress is the result of NO increase via iNOS, and that the promotion of osteoclast differentiation and fusion after the release from mechanical stress is related to the NFATc family genes, whose expression remained constant during mechanical stress but was up-regulated after the release from mechanical stress.

\section{Introduction}

Bone homeostasis is maintained by the balance between bone formation and bone resorption (1). Tipping the balance in favor of osteoclasts leads to diseases with a low bone mass, including osteoporosis, whereas impaired osteoclastic bone resorption results in diseases with high bone mass, including osteopetrosis (2). The relationship between the immune and skeletal systems has gradually become clearer $(3,4)$. Accumulating data on the molecular mechanisms of osteoclast differentiation have demonstrated that the receptor activator of nuclear factor- $\kappa \mathrm{B}$ (RANK)-ligand (RANKL) pathway and the macrophage colony-stimulating factor (M-CSF) are necessary and sufficient for the differentiation of osteoclasts $(5,6)$. In addition, the role of the nuclear factor of activated T cells cytoplasmic (NFATc) 1 gene, a master switch for the regulation of the terminal differentiation of osteoclasts which functions downstream of RANKL, has been demonstrated both in vitro and in vivo (7-9). NFATc1 expression is dependent on both the TRAF6/NF- $\kappa \mathrm{B}$ and the c-Fos pathways. The NFAT family of transcription factors was originally discovered in $\mathrm{T}$ cells of the immune system, but they are involved in the regulation of various biological systems including the $\mathrm{Ca}^{2+}$-regulated pathway (9).

Mechanical stress can largely affect this balanced bone metabolism. Bone adjusts to load by changing its mass, shape or microarchitecture (10). It has been reported that removing the load results in bone loss (11), whereas application of a load causes bone apposition (12). However, the precise mechanism by which loading is transduced into cellular control of bone remodeling is unknown. A number of studies in vitro and in vivo have described the effects of mechanical stress on the bone cells. To investigate the influence of mechanical stress on the cells, several in vitro models have been established with varying methods, including sheer stress 
(fluid flow) $(13,14)$ compressive $(15,16)$ and tensile force $(17)$, hydrostatic pressure (18), microgravity (19), and so on $(20,21)$. Furthermore, in addition to an in vivo experimental system including transgenic mice, many studies have indicated the effect of mechanical stress on the bone's adaptive remodeling behavior in osteocytes (22), osteoblasts (23) and in a co-culture between osteoblasts and osteoclasts (24). Several studies have shown that nitric oxide (NO) acts as a mediator of mechanically induced bone remodeling $(10,25,26)$. However, the direct effect of mechanical stress on the behavior of osteoclasts (27) has only been investigated in a few studies and there is limited information on the effects of the release from mechanical stress, which thus became the focus of the present study.

The aim of this study was to elucidate which osteoclast differentiation genes are altered in response to the release from mechanical stress and whether such an alteration is mediated by loading-induced NO production. To validate that NO may modulate the differentiation of osteoclasts in response to mechanical stress, we inhibited the release of NO by an NO synthetase (NOS) inhibitor.

\section{Materials and methods}

Cell culture and the Flexercell tension system. We used the murine monocyte/macrophage cell line RAW264.7 cells (RAW cells) (ATCC no. TIB-71 ${ }^{\mathrm{TM}}$, USA) as osteoclast precursors. The cells were grown to subconfluence in $100 \mathrm{~mm}$ standard dishes $\left(\right.$ Falcon $^{\mathrm{TM}}$, Becton-Dickinson, Franklin Lakes, NJ, USA) with Dulbecco's modified Eagle's medium (DMEM) (Wako Pure Chemical, Japan) supplemented with $10 \%$ heat-inactivated fetal bovine serum (FBS) (Invitrogen, MD, USA) and kanamycin sulfate (Meiji Seika, Japan) at $37^{\circ} \mathrm{C}$ in a humidified atmosphere of $95 \%$ air and $5 \% \mathrm{CO}_{2}$. Subsequently, RAW cells $\left(3 \times 10^{4}\right.$ cells/well) were transferred to Flexcell 6-well culture plates with a type I collagen-coated and flexible-bottomed well (Flexcell Corp., Hillsborough, NC, USA), and cultured with $\alpha$-MEM (Wako Pure Chemical) supplemented with $10 \%$ heat-inactivated FBS, $50 \mathrm{ng} / \mathrm{ml}$ RANKL (Oriental Yeast, Japan), 2 mM L-alanyl-L-glutamine (Sigma-Aldrich, St. Louis, MO, USA), $284 \mu \mathrm{M}$ L-ascorbic acid 2-phosphate (Sigma-Aldrich) and $66.7 \mu \mathrm{g} / \mathrm{ml} \mathrm{kanamycin}$ sulfate at $37^{\circ} \mathrm{C}$ in a humidified atmosphere of $95 \%$ air and $5 \% \mathrm{CO}_{2}$. RAW cells differentiated into TRAP-positive multinucleated osteoclastic cells in the presence of RANKL (27). Mechanical stress was applied to RAW cells with a Flexercell tension system (28). In this device, a flexible silicon membrane is stretched across a loading post by the application of a vacuum pressure. The experimental regimens used in this study delivered $10 \%$ elongation at 30 cycles per $\min (0.5 \mathrm{~Hz})(29)$. Mechanical stress was applied for a period of 48 h over 3-4 days (3-4 MS). After the termination of the mechanical stress application, culturing of the RAW cells continued for the indicated periods. The culture medium was replaced on days $2,3,5$ and 7 of culturing.

Tartrate-resistant acid phosphatase (TRAP) staining. The cells cultured for a given period were fixed with $10 \%$ neutral formalin. They were then washed with distilled water and stained in the TRAP staining solution ( $\mathrm{pH}$ 5.0) with Fast Red Violet LB salt (Sigma-Aldrich) (30). TRAP-positive
Table I. The assay ID of the primers used.

\begin{tabular}{ll}
\hline Gene & Assay ID \\
\hline RANK & Mm00475698_m1 \\
$c$-fms & Mm00432689_m1 \\
OSCAR & Mm00558663_m1 \\
NFATc1 & Mm00479445_m1 \\
NFATc2 & Mm00477776_m1 \\
NFATc3 & Mm01249194_m1 \\
IFN- $\beta$ & Mm00439546_s1 \\
iNOS & Mm00440485_m1 \\
nNOS & Mm00435175_m1 \\
TRAP & Mm00475698_m1 \\
CTR & Mm00432271_m1 \\
MMP-9 & Mm00432271_m1 \\
cath-k & Mm00484036_m1 \\
ClC7 & Mm00442400_m1 \\
ATP6i & Mm00469395_g1 \\
DC-STAMP & Mm01168058_m1 \\
GAPDH & Mm99999915_g1 \\
\hline &
\end{tabular}

cells with $>2$ nuclei under a light microscope were counted as osteoclasts. Osteoclasts with $>2$ nuclei were distinguished from large osteoclasts with $\geq 8$ nuclei (27). The control culture was grown under the same condition without mechanical stress. The counted range was $1 \mathrm{~cm}^{2}$ dividing the circle in a fan-shape.

Reverse transcription-polymerase chain reaction and real time PCR. Total RNA was extracted using the TRIzol reagent isolation kit (Invitrogen) (31). Reverse transcription was performed with $1 \mu \mathrm{g}$ of total RNA in a total volume of $20 \mu 1$ per reaction. cDNA was amplified by Rever Tra Ace- $\alpha$ FSK-101 (Toyobo, Japan). The following primers (Applied Biosystems, USA) were used: RANK, M-CSF receptor (c-fms), osteoclast-associated receptor (OSCAR), TRAP, matrix metalloproteinase-9 (MMP-9), cathepsin-K (cath-k), calcitonin receptor (CTR), dendritic cell-specific transmembrane protein (DC-STAMP) ATPase $\mathrm{H}^{+}$transporting vacuolar proton pump member I (ATP6i), chloride channel-7 (ClC7), NFATc1, NFATc2, NFATc3, inducible NOS (iNOS) neuronal NOS (nNOS) and interferon- $\beta$ (IFN- $\beta$ ) as shown in Table I. As an internal control for RNA quantity, the same cDNA was amplified using primers specific for glyceraldehyde-3-phosphate dehydrogenase (GAPDH) mRNA. All amplification reactions were performed in triplicate. The ABI Prism 7300 sequence detection system (Applied Biosystems) (32) was used for realtime PCR analysis. At the end of each PCR run, the data were automatically analyzed by the system and amplification plots were obtained. The $\Delta \Delta \mathrm{Ct}$ method was used to calculate the real-time PCR results (amount of target $=2^{-\Delta \Delta C t}$ ).

Inhibition of nitric oxide production. NO release was inhibited by the addition of $\mathrm{N}^{\mathrm{G}}$-Nitro-L-arginine methyl ester, hydrochloride (L-NAME), (Dojindo, Japan). L-NAME was 

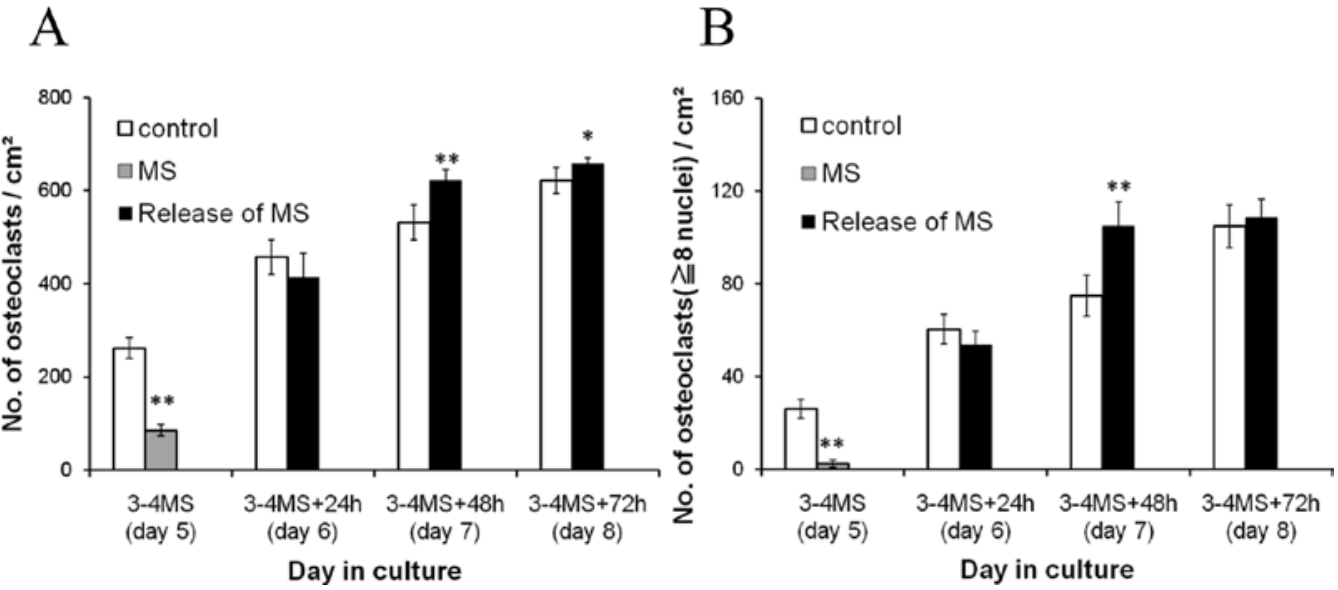

Figure 1. The number of TRAP-positive multinucleated osteoclasts was suppressed with mechanical stress, and then was increased after mechanical stress release through osteoclast differentiation. Mechanical stress was applied for $48 \mathrm{~h}$ over 3-4 days, but not to control. After the release from mechanical stress, TRAP-positive multinucleated osteoclasts were counted until $72 \mathrm{~h}$ every $24 \mathrm{~h}$. (A) The number of TRAP-positive multinucleated cells. (B) The number of large osteoclasts with $\geq 8$ nuclei. Magnification $x 100$. Results are shown as mean $\pm \mathrm{SD}(\mathrm{n}=12)$.

dissolved in PBS and added to the culture medium (final concentration, $100 \mu \mathrm{M})(33)$.

Nitrite and nitrate measurements. The $\mathrm{NO}$ metabolite $\left(\mathrm{NO}_{2}\right.$ and $\mathrm{NO}_{3}$ ) levels were analyzed in the conditioned medium by means of a colorimetric assay kit: $\mathrm{NO}_{2} / \mathrm{NO}_{3}$ Assay kit-C II (Dojindo). Samples were read at $540 \mathrm{~nm}$ with a Spectra Microplate Auto reader (Bio-Rad Model 680, Hercules, CA, USA).

Statistics. All results are given as mean \pm SD. Comparisons between 2 groups were analyzed using the 2-tailed unpaired Student's t-test. P-values of $<0.05$ were considered statistically significant.

\section{Results}

The number of osteoclasts increased rapidly after the release from mechanical stress. The effect of mechanical stress and the release from mechanical stress on osteoclast differentiation was examined. Mechanical stress was applied for $48 \mathrm{~h}$ over 3-4 days. The number of TRAP-positive multinucleated osteoclasts was assessed every $24 \mathrm{~h}$ after the release from mechanical stress up to $72 \mathrm{~h}$. The control group was grown under the same conditions without mechanical stress. Mechanical stress caused suppression in the number of osteoclasts to less than one third of that in the control group (Fig. 1A). The mechanical stress-induced decrease in the number of large osteoclasts with $\geq 8$ nuclei (Fig. 1B) was more pronounced than that in the osteoclasts with $>2$ nuclei. On the other hand, osteoclast differentiation and fusion were rapidly promoted after the release from mechanical stress and exceeded the control group $48 \mathrm{~h}$ after the release from it (Fig. 1).

The effects of mechanical stress application and its release on the expression of osteoclast differentiation genes. We investigated how the expression of osteoclast differentiation genes was altered by mechanical stress application and its release, using a real-time PCR analysis (Fig. 2) for osteoclast specific genes (TRAP, CTR, MMP-9, cath-k, ClC7, ATP6i), which are differentiation markers detected after the formation of mature osteoclasts, and for DC-STAMP. The expression of these genes was decreased in the mechanical stress group, and increased after the release from mechanical stress up to $48 \mathrm{~h}$ compared to the control group. The expression of iNOS mRNA was increased in the mechanical stress group, and then decreased after the release from mechanical stress compared to the control group. On the other hand, the expression of nNOS mRNA was slightly decreased in the mechanical stress group, and remained fairly constant after the release from mechanical stress compared to the control group. We could not detect eNOS mRNA expression in these osteoclastic RAW cells (data not shown). The expression of IFN- $\beta$ mRNA was significantly decreased in the mechanical stress group, and then continued decreasing after the release from mechanical stress compared to the control group. The mRNA expression of NFATc family gene members was not altered during mechanical stress application, and was up-regulated after the release from mechanical stress and up to $48 \mathrm{~h}$ compared to the control group. The expression of RANK mRNA was unchanged and that of OSCAR mRNA was increased $48 \mathrm{~h}$ after the release from mechanical stress, although they were slightly decreased in the mechanical stress group compared to the control group. The expression of c-fms mRNA was increased $24 \mathrm{~h}$ after the release from mechanical stress, although it was not significantly difference in the mechanical stress group compared to the control group.

Mechanical stress suppressed osteoclastogenesis and increased NO production and the NOS inhibitor, L-NAME, reduced the effect of mechanical stress. A significant increase in $\mathrm{NO}$ production was observed in the mechanical stress group. The concentration of $\mathrm{NO}_{2}{ }^{-} / \mathrm{NO}_{3}{ }^{-}$in the conditioned medium with the mechanical stress showed an approximately 2 -fold increase compared to the control group (Fig. 3). The concentration of $\mathrm{NO}_{2}^{-} / \mathrm{NO}_{3}^{-}$in the conditioned medium with the mechanical stress and L-NAME showed a decrease compared with the mechanical stress only group (Fig. 3). 

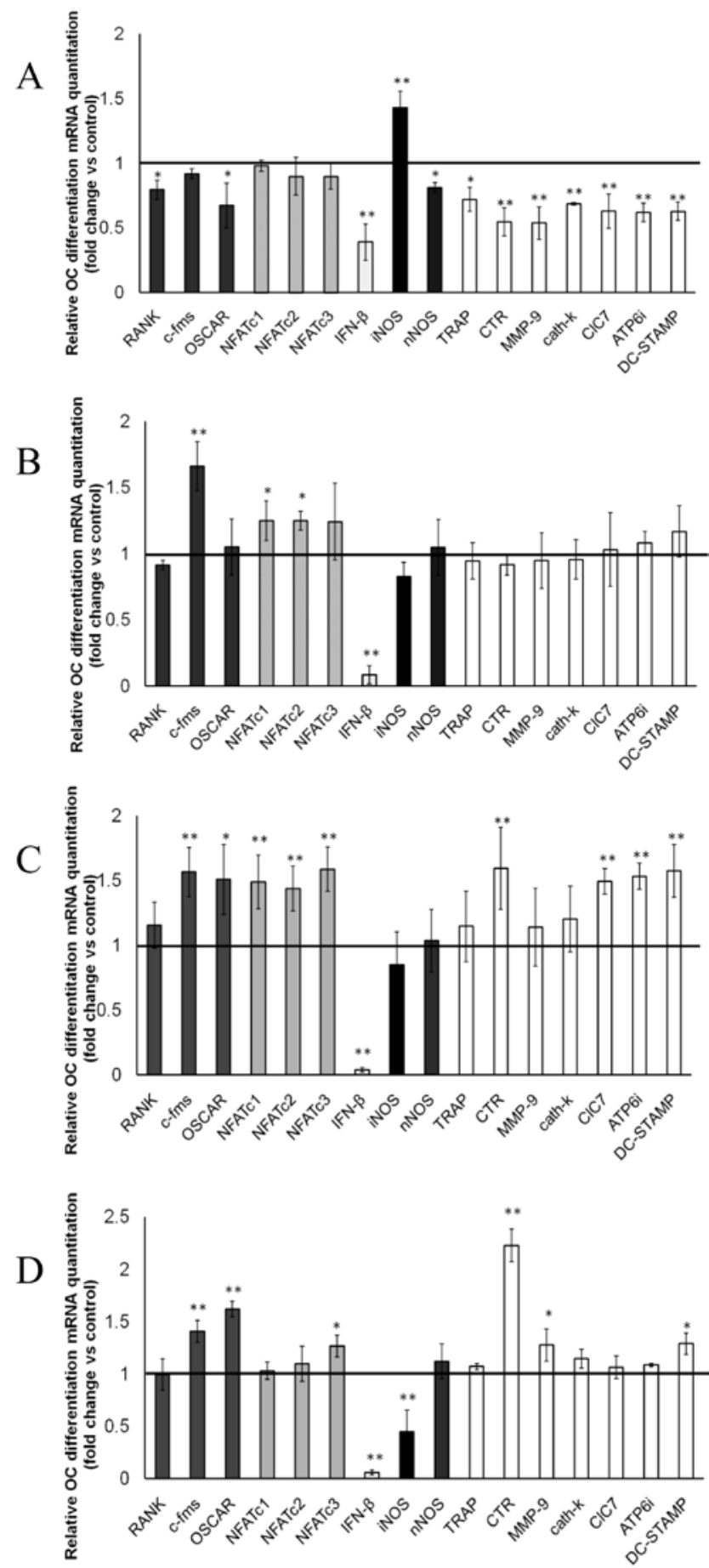

Figure 2. Effects of mechanical stress application and release on the expressions of RANK, c-fms, OSCAR, the NFATc family, iNOS, nNOS, IFN- $\beta$, TRAP, CTR, MMP-9, cath-k, ClC7, ATP6i and DC-STAMP. Real-time PCR analysis was performed with indicated primers in the mechanical stress group (A), and at $24 \mathrm{~h}(\mathrm{~B}), 48 \mathrm{~h} \mathrm{(C)}$ and $72 \mathrm{~h}(\mathrm{D})$ after the release of mechanical stres. Results are shown as fold-changes or relative quantitation of target expression ( $2^{-\Delta \Delta C t}$ method) relative to control after normalization against GAPDH expression and as mean $\pm \mathrm{SD}(\mathrm{n}=6)$. The control group which was cultured for the same days without mechanical stress was defined as the standard $(n=1)$.

L-NAME had no effect on differentiation under basal conditions in osteoclasts (Fig. 4A). Addition of L-NAME to the culture medium with mechanical stress partially blocked the effect of mechanical stress on osteoclast differentiation and

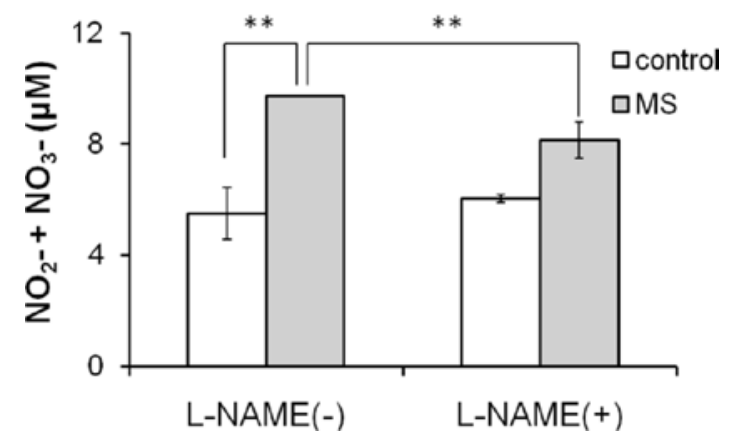

Figure 3. Mechanical stress (MS) increased nitric oxide (NO) production and L-NAME reduced the effect of mechanical stress on osteoclastogenesis in RAW cells. Mechanical stress was applied to RAW cells with a Flexercell tension system for a period of $48 \mathrm{~h}$ over 3-4 days (grey bar), but not to the control (white bar) in the absence (-) or in the presence (+) of L-NAME. Total $\mathrm{NO}_{2}^{-}+\mathrm{NO}_{3}^{-}$accumulated in the conditioned medium were shown as NO production. The data shown are representative of the results obtained in three experiments. Results are shown as the mean $\pm S D(n=3)$.

fusion. The number of both osteoclasts and large osteoclasts induced by mechanical stress application and L-NAME treatment was increased approximately 2-fold compared to the mechanical stress only group (Fig. 4).

The effect of mechanical stress and L-NAME on the expressions of osteoclast differentiation genes. In order to confirm the affect of nitrite accumulation induced by mechanical stress and L-NAME treatment, we employed real-time PCR (Fig. 5). The expression of osteoclast specific gene mRNAs (TRAP, CTR, MMP-9, cath-k, ClC7, ATP6i) and DC-STAMP mRNA was increased with L-NAME treatment in the mechanical stress group compared to the mechanical stress only group. The expression of iNOS mRNA showed a tendency to decrease, however, the difference was not statistically significant. The expression IFN- $\beta$ mRNA significantly increased with L-NAME treatment in the mechanical stress group compared with the mechanical stress only group. The expression of RANK, c-fms and NFATc1 mRNA was unchanged between the two groups.

\section{Discussion}

Application of mechanical stress on bone tissue generates extracellular matrix deformation, and contributes to the regulation of bone modeling and remodeling. Thereby, mechanical stress is translated into mechanical signals. Several studies have shown that these mechanical signals could adaptively change the functions of osteoblasts, osteocytes and bone stromal cells $(10,34,35)$. In contrast, very few studies have focused on the direct effects of mechanical stress on osteoclast RAW cells. Furthermore, many studies have shown that loading is necessary to generate an anabolic effect in the bone (36), and that the anabolic response to loading declines soon after loading is initiated and mechanosensitivity is increased by the insertion of rest periods (37). However, the precise consequences of the removal of mechanical signals from bone tissue are currently difficult to predict in vivo, because they are dependent on the interactions between genetics, gender and the specific anatomical site. The effects 

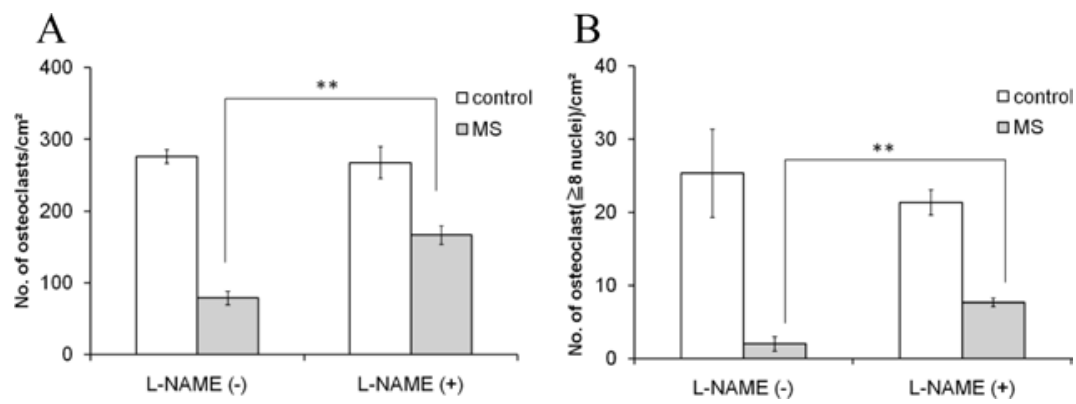

Figure 4. Suppression of the number of TRAP-positive multinucleated osteoclasts induced by mechanical stress (MS) was partially recovered by treatment with the NOS inhibitor, L-NAME. RAW cells were cultured in Flexcell 6-well plates with RANKL in the absence (-) or in the presence (+) of L-NAME. (A) The number of TRAP-positive multinucleated cells. (B) The number of large osteoclasts with $\geq 8$ nuclei. Magnification x100. Results are shown as the mean $\pm \operatorname{SD}(n=3)$.
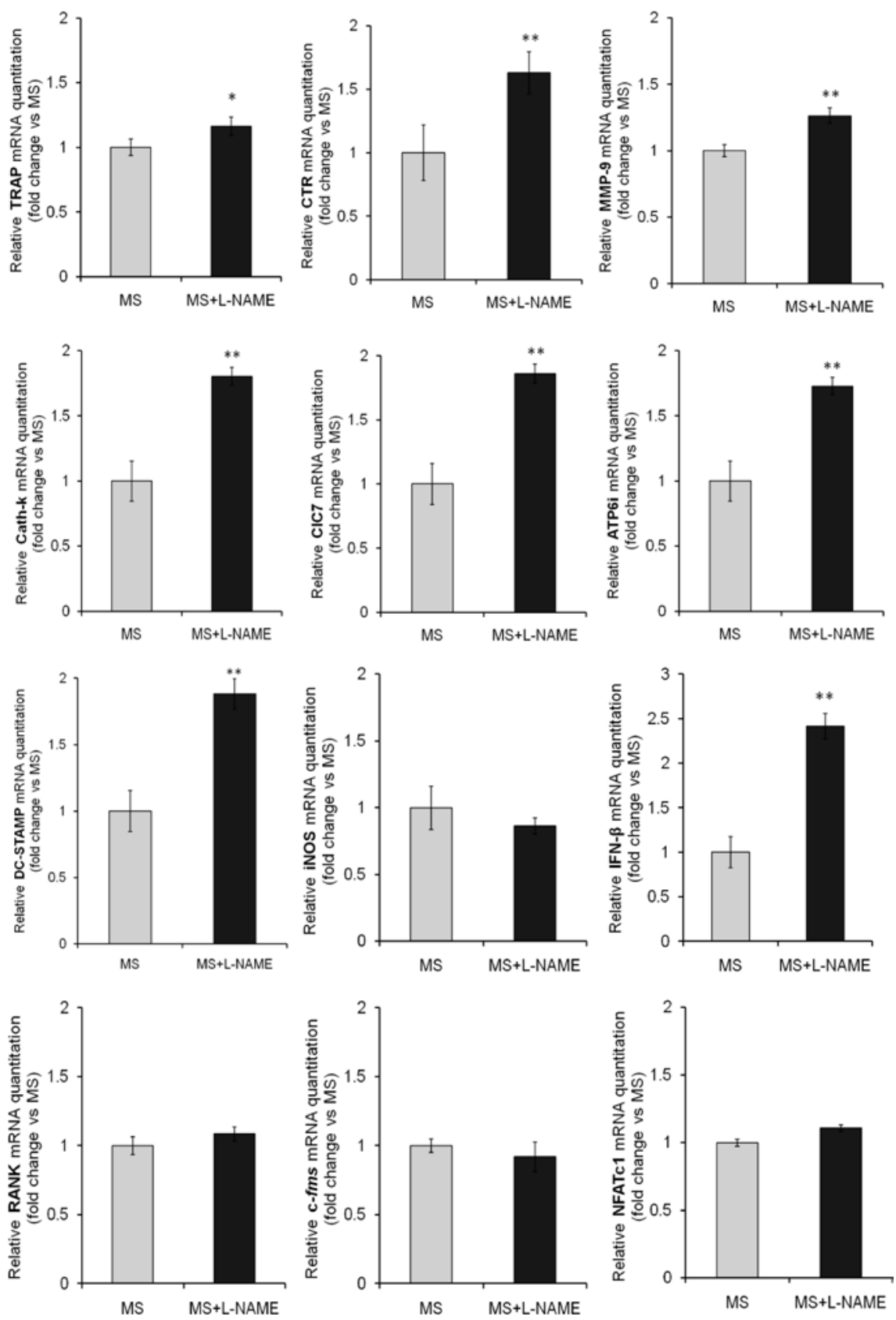

Figure 5. Effect of mechanical stress together with L-NAME on the expression of TRAP, CTR, MMP-9, cath-k, ClC7, ATP6i, DC-STAMP, IFN- $\beta$, iNOS, RANK, c-fms and NFATc1. RAW cells were cultured in Flexcell 6-well plates with mechanical stress in the absence (grey bar) or in the presence (black bar) of L-NAME. Results are presented as the mean $\pm S D(n=3)$ of fold-changes or relative quantitation of target expression $\left(2^{-\Delta A C t}\right.$ method) relative to the mechanical stress group after normalization against GAPDH expression. The mechanical stress group were defined as the standard (=1). MS, mechanical stress group; MS + L-NAME, treatment of L-NAME with MS. 
of release from mechanical stress may be as important as the effects of mechanical stress. For this reason, in the present study we have evaluated the effects of mechanical stress applied with the Flexercell tension system and of the release from mechanical stress on osteoclastogenesis and further investigated the molecular mechanisms involved.

Previously, we have shown that mechanical stress directly suppresses osteoclast differentiation suggesting delayed differentiation in RAW cells (27). In the present study, we have demonstrated that the suppression of osteoclast differentiation and fusion with mechanical stress is related to the increase of NO via iNOS, and that the promotion of osteoclast differentiation and fusion after the release from mechanical stress is related to the NFATc family genes, whose expression remained constant during mechanical stress but was up-regulated after the release from mechanical stress. NO is a small, diffusible, diatomic free radical involved in several biological processes, including the regulation of bone formation, resorption, remodeling, mechanotransduction and repair in physiological or pathophysiological conditions. NO is an important regulator of the response of the bone to mechanical stress, and is produced through the activities of constitutive eNOS or iNOS (38). Several in vitro studies have shown that NO rapidly increases in response to mechanical stress in bone cells (39). NO mediates adaptive bone formation, protects osteocytes against apoptosis and mediates osteoclast activity (26). High levels of NO reduce osteoclast activity, while the inhibition of NO production increases osteoclastogenesis and osteoclast activity (40). iNOS has been detected in osteocytes, osteoclasts and chondrocytes and is expressed in response to inflammatory cytokines. Once induced, iNOS produces large amounts of NO (38). Thus, our results together with the previous findings may further contribute to the understanding of the relationship between mechanical stress and NO in osteoclasts. Indeed, in the present study we have shown that NO via iNOS is increased with mechanical stress application and suppresses osteoclast differentiation and fusion, although we could not detect eNOS mRNA expression in these osteoclastic RAW cells. IFN- $\beta$ has been identified as a RANKL-stimulated autocrine negative feedback inhibitor that limits osteoclastogenesis and triggers iNOS/NO as an important negative feedback signal during osteoclastogenesis (41). In this study, the expression of IFN- $\beta$ mRNA is decreased during mechanical stress and after the release from mechanical stress and is increased with L-NAME treatment. This result might contribute, at least in part, to the abovementioned negative feedback signal.

NFATc1-deficient embryonic stem cells fail to differentiate into osteoclasts in response to RANKL stimulation and ectopic expression of NFATc1 causes precursor cells to undergo efficient differentiation without RANKL signaling, indicating that NFATc1 is a master switch for regulating the terminal differentiation of osteoclasts, functioning downstream of RANKL (7). Transcriptional activation of NFATc1 in osteoclasts is mediated by the RANKL/TRAF6/c-Fos signaling pathway because NFATc1 rescues osteoclastogenesis in precursors lacking c-Fos (3). In the present study, the mRNA expression of NFATc family genes was constant in spite of the suppression of osteoclast-specific genes, and was up-regulated after the release from mechanical stress. This result suggests that homeostasis was maintained under conditions of mechanical stress through various courses of action.

With respect to the osteoclast specific genes (TRAP, CTR, MMP-9, cath-k, ClC7, ATP6i) and DC-STAMP which are essential for cell to cell fusion in osteoclasts (42), the expression of these mRNA was decreased with mechanical stress, and then increased after the release from mechanical stress up to $48 \mathrm{~h}$ compared to the control group, correlating with the number of both osteoclasts and large osteoclasts. However, $72 \mathrm{~h}$ after the release from mechanical stress, CTR whose expression inhibits osteoclast activity both in vitro and in vivo (43) was increased. This result might stand opposite to the osteoclasts which increased rapidly after the release from mechanical stress. Expression of the c-fms receptor is necessary for osteoclastogenesis and increased $24 \mathrm{~h}$ after the release from mechanical stress, although it was not significantly different in the mechanical stress group compared to the control group. RAW cells did not require exogenous M-CSF for RANKL-induced osteoclast formation. The capability of producing M-CSF in response to RANKL may enable RAW cells to differentiate into osteoclasts in the presence of RANKL alone (44). This capability may be different between physiological osteoclast precursors and RAW cells. OSCAR is critical for osteoclast differentiation. OSCAR mRNA expression was increased $48 \mathrm{~h}$ after the release from mechanical stress, although it was slightly decreased in the mechanical stress group compared to the control group. FcR $\gamma$-mediated signal transduction by OSCAR is involved in osteoclast differentiation (45). FcR $\gamma$-associated receptors are only activated by osteoblasts. In the RANKL/M-CSF system DAP12-associated receptors may be activated by endogenous ligands provided by osteoclast precursor cells themselves. DAP12 and FcR $\gamma$ compensated for each other in the activation of the ITAM signal, and the cooperation of the RANKL and ITAM signals is essential for NFATc1 induction and osteoclastogenesis (9). Hence c- $f m s$ and OSCAR mRNA expression may be associated only to a small extent with the effects of mechanical stress on the osteoclastogenesis of RAW cells.

In summary, we have shown that the effects of the release from mechanical stress may be as important as the effects of mechanical stress on osteoclastogenesis. Our aim was to investigate the osteoclast differentiation genes which are altered in response to the release from mechanical stress applied with the Flexercell tension system. We have found that the suppression of osteoclast differentiation and fusion induced by mechanical stress resulted from the increase of NO via iNOS, and that the promotion of osteoclast differentiation and fusion after the release from mechanical stress is related to the NFATc family genes, whose expression remained constant during mechanical stress but was up-regulated after the release from mechanical stress.

\section{Acknowledgements}

We are grateful to Dr N. Suzuki and Dr T. Nakai for technical advice and support. This study was supported in part by the Japan Society for the Promotion of Science Grant-in-Aid for Scientific Research (C) (grant no. 22592274 to Y.Y. and no. 21592584 to T.K.) and a Grant-in-Aid for Young Scientists (B) (grant no. 22792032 to Y.T.). 


\section{References}

1. Karsenty G and Wagner EF: Reaching a genetic and molecular understanding of skeletal development. Dev Cell 2: 389-406, 2002.

2. Teitelbaum SL and Ross FP: Genetic regulation of osteoclast development and function. Nat Rev Genet 4: 638-649, 2003.

3. Nakashima $\mathrm{T}$ and Takayanagi $\mathrm{H}$ : Osteoclasts and the immune system. J Bone Miner Metab 27: 519-529, 2009.

4. Nakashima $T$ and Takayanagi H: Osteoimmunology: crosstalk between the immune and bone systems. J Clin Immunol 29: 555-567, 2009.

5. Yasuda H, Shima N, Nakagawa N, et al: Osteoclast differentiation factor is a ligand for osteoprotegerin/osteoclastogenesis-inhibitory factor and is identical to TRANCE/RANKL. Proc Natl Acad Sci USA 95: 3597-3602, 1998.

6. Yoshida H, Hayashi S, Kunisada T, et al: The murine mutation osteopetrosis is in the coding region of the macrophage colony stimulating factor gene. Nature 345: 442-444, 1990.

7. Takayanagi $\mathrm{H}$, Kim S, Koga $\mathrm{T}$, et al: Induction and activation of the transcription factor NFATc1 (NFAT2) integrate RANKL signaling in terminal differentiation of osteoclasts. Dev Cell 3 : 889-901, 2002

8. Asagiri M, Sato K, Usami T, et al: Autoamplification of NFATc1 expression determines its essential role in bone homeostasis. J Exp Med 9: 1261-1269, 2005.

9. Takayanagi H: Mechanistic insight into osteoclast differentiation in osteoimmunology. J Mol Med 83: 170-179, 2005.

10. Ehrlich PJ and Lanyon LE: Mechanical strain and bone cell function: a review. Osteoporos Int 13: 688-700, 2002.

11. Schneider V, Oganov V, LeBlanc A, et al: Bone and body mass changes during space flight. Acta Astronaut 36: 463-466, 1995

12. Srinivasan S, Weimer DA, Agans SC, Bain SD and Gross TS Low-magnitude mechanical loading becomes osteogenic when rest is inserted between each load cycle. J Bone Miner Res 17: 1613-1620, 2002.

13. Mehrotra M, Saegusa M, Wadhwa S, Voznesensky O, Peterson D and Pilbeam C: Fluid flow induces RANKL expression in primary murine calvarial osteoclasts. J Cell Biochem 98 1271-1283, 2006

14. Tan SD, de Vries TJ, Kuijpers-Jagtman AM, Semeins CM, Everts V and Klein-Nulend J: Osteocytes subjected to fluid flow inhibit osteoclast formation and bone resorption. Bone 41: 745-751, 2007.

15. Ichimiya H, Takahashi $\mathrm{T}$, Ariyoshi $\mathrm{W}$, et al: Compressive mechanical stress promotes osteoclast formation through RANKL expression on synovial cells. Oral Surg Oral Med Oral Pathol Oral Radiol Endod 103: 334-341, 2007.

16. Liu J, Zhao Z, Zou L, et al: Pressure-loaded MSCs during early osteodifferentiation promote osteoclastogenesis by increase of RANKL/OPG ratio. Ann Biomed Eng 37: 794-802, 2009.

17. Kanzaki H, Chiba M, Sato A, Miyagawa A, Arai K, Nukatsuka S and Mitani $\mathrm{H}$ : Cyclical tensile force on periodontal ligament cells inhibits osteoclastogenesis through OPG induction. J Dent Res 85: 457-462, 2006

18. Rubin J, Biskobing D, Fan X, Rubin C, McLeod K and Taylor WR: Pressure regulates osteoclast formation and MCSF expression in marrow culture. J Cell Physiol 170: 81-87, 1997.

19. Makihira S, Kawahara Y, Yuge L, Mine Y and Nikawa H: Impact of the microgravity environment in a 3-dimensional clinostat on osteoblast- and osteoclast-like cells. Cell Biol Int 32: 1176-1181, 2008

20. Kadow-Romacker A, Hoffmann JE, Duda G, Wildemann B and Schmidmaier G: Effect of mechanical stimulation on osteoblastand osteoclast-like cells in vitro. Cells Tissues Organs 190 61-68, 2009.

21. Kurata K, Uemura T, Nemoto A, et al: Mechanical strain effect on bone-resorbing activity and messenger RNA expressions of marker enzymes in isolated osteoclast culture. J Bone Miner Res 16: 722-730, 2001

22. Robling AG, Niziolek PJ, Baldridge LA, et al: Mechanical stimulation of bone in vivo reduces osteocyte expression of Sost/ sclerostin. J Biol Chem 283: 5866-5875, 2008.

23. Inoue $D, K$ Kido $S$ and Matsumoto T: Transcriptional induction of FosB/DeltaFosB gene by mechanical stress in osteoblasts. J Bio Chem 279: 49795-49803, 2004.
24. Rubin J, Murphy TC, Fan X, Goldschmidt M and Taylor WR Activation of extracellular signal-regulated kinase is involved in mechanical strain inhibition of RANKL expression in bone stromal cells. J Bone Miner Res 17: 1452-1460, 2002.

25. Rahnert J, Fan X, Case N, Murphy TC, Grassi F, Sen B and Rubin J: The role of nitric oxide in the mechanical repression of RANKL in bone stromal cells. Bone 43: 48-54, 2008.

26. Tan SD, Kuijpers-Jagtman AM, Semeins CM, et al: Fluid shear stress inhibits TNFalpha-induced osteocyte apoptosis. J Dent Res 85: 905-909, 2006.

27. Suzuki N, Yoshimura Y, Deyama Y and Suzuki K: Mechanical stress directly suppresses osteoclast differentiation in RAW264.7 cells. Int J Mol Med 21: 291-296, 2008.

28. Vande Geest JP, Di Mrtino ES and Vorp DA: An analysis of the complete strain field within Flexercell membranes. J Biomech 37: 1923-1928, 2004

29. Nakai T, Yoshimura Y, Deyama Y and Suzuki K: Mechanical stress up-regulates RANKL expression via the VEGF autocrine pathway in osteoblastic MC3T3-E1 cells. Mol Med Rep 2: 229-234, 2009.

30. Takeyama S, Yoshimura Y, Deyama Y, Sugawara Y, Fukuda H, and Matsumoto A: Phosphate decreases osteoclastgenesis in coculture of osteoblast and bone marrow. Biochem Biophys Res Commun 282: 798-802, 2001

31. Nakamura K, Deyama Y, Yoshimura Y, Suzuki K and Morita M: Toll-like receptor 3 ligand-induced antiviral response in mouse osteoblastic cells. Int J Mol Med 19: 771-775, 2007.

32. Aguirre JI, Plotkin LI, Gortazar AR, et al: A novel ligandindependent function of the estrogen receptor is essential for osteocyte and osteoblast mechanotransduction. J Biol Chem 282: 25501-25508, 2007.

33. Pitsillides AA, Rawlinson SC, Suswillo RF, Bourrin S, Zaman G and Lanyon LE: Mechanical strain-induced NO production by bone cells: a possible role in adaptive bone (re)modeling? FASEB J 9: 1614-1622, 1995

34. Rubin J, Rubin C and Jacobs CR: Molecular pathways mediating mechanical signaling in bone. Gene 367: 1-16, 2006.

35. Galli C, Passeri G and Macaluso GM: Osteocytes and WNT: the mechanical control of bone formation. J Dent Res 89: 331-343, 2010.

36. Judex S, Gupta S and Rubin C: Regulation of mechanical signals in bone. Orthod Craniofac Res 12: 94-104, 2009.

37. Robling AG, Burr DB and Turner $\mathrm{CH}$ : Recovery periods restore mechanosensitivity to dynamically loaded bone. J Exp Biol 204: 3389-3399, 2001

38. Van't Hof RJ and Ralston SH: Nitric oxide and bone. Immunology 103: 255-261, 2001.

39. Bacabac RG, Smit TH, Mullender MG, Dijcks SJ, Van Loon JJ and Klein-Nulend J: Nitric oxide production by bone cells is fluid shear stress rate dependent. Biochem Biophys Res Commun 315: 823-829, 2004

40. Wimalawansa SJ: Nitric oxide and bone. Ann NY Acad Sci 1192: 391-403, 2010.

41. Zheng H, Yu X, Osdoby PC and Osdby P: RANKL stimulates iNOS expression and NO production in developing osteoclasts: an autocrine negative feedback mechanism triggered by RANKL-induced IFN- $\beta$ via NF- $\kappa$ B that restrains osteoclastogenesis and bone. J Biol Chem 281: 15809-15820, 2006.

42. Yagi M, Miyamoto T, Sawatani Y, et al: DC-STAMP is essential for cell-cell fusion in osteoclasts and foreign body giant cells. J Exp Med 202: 345-351, 2005.

43. Mancini L, Moradi-Bidhendi N, Brandi ML, Perretti M and MacIntyre I: Modulation of the effects of osteoprotegerin (OPG) ligand in a human leukemic cell line by OPG and calcitonin. Biochem Biophys Res Commun 279: 391-397, 2000.

44. Islam S, Hassan F, Tumurkhuu G, et al: Receptor activator of nuclear factor-kappa B ligand induces osteoclast formation in RAW 264.7 macrophage cells via augmented production of macrophage-colony-stimulating factor. Microbiol Immunol 52: 585-590, 2008

45. Ishikawa S, Arase N, Suenaga T, et al: Involvement of FcRgamma in signal transduction of osteoclast-associated receptor (OSCAR). Int Immunol 16: 1019-1025, 2004. 